\title{
Key Technologies on YAG Laser Engraving Ceramic Anilox Roll
}

\author{
Hongcai Wang, Yang Wang \\ School of Mechatronics Engineering \\ Harbin Institute of Technology \\ Harbin, China \\ whongc@sina.com
}

\author{
Hongcai Wang, Mingjiang Yang \\ Key Laboratory of Mechanics in Advanced Manufacturing \\ Institute of Mechanics, Chinese Academy of Sciences \\ Beijing, China
}

\begin{abstract}
As the heart of Flexography,laser engraving ceramic anilox roll requires that the diameter and depth of each cell is changeable, and the distribution of cell should be $30^{\circ}$-hexagon, $45^{\circ}$-quadrangle or $60^{\circ}$-hexagon. By modulating the laser cavity gain from step way to gradual chang way, the wave modulating technology of high repetition light pulse solves the problem of increasing absorbency of ceramic to YAG laser. By engraving the same cell with grouped light pulses, the technology of high repetition and grouped pulses solves the problem of engraving high depth cell. By detecting with corder and processing using alterable divider coefficient,the technology of tow-dimension controllable distribution solves the problems of orientation and distribution of each cell. These three YAG laser texturing technologies above-mentioned are the key technologies to realize YAG laser engraving ceramic anilox roll.
\end{abstract}

Keywords-laser technique; ceramic anilox roll; laser texturing; tow-dimension controllable distribution

\section{I. 引言}

柔版印刷由于使用水基油墨、UV 油墨和醇溶剂油墨 等环保油墨, 在包装印刷行业得到了广泛的应用 ${ }^{[1]}$ 。网纹 辊是柔印机的传墨辊，其表面制有凹下的网穴，用于控制 传墨量。网纹辊可以精确控制墨层厚度, 为提高印品质量 提供了重要保证, 被称为柔版印刷的心脏 ${ }^{[2]}$ 。

早期使用的网纹辊是金属网纹辊。后来出现了镀铬金 属网纹辊和喷涂陶瓷网纹辊。受雕刻方法的限制, 以上三 种网纹辊的雕刻线数最高不超过 500 线/英寸, 影响了印 刷质量。

1984 年 $\mathrm{CO}_{2}$ 激光雕刻陶瓷网纹辊被正式采用, 其雕 刻线数最高可达 1200 线/英寸, 印刷质量得到了很大的提 升。激光雕刻陶瓷网纹辊与传统网纹辊相比, 其寿命有很 大的提高, 换辊周期由 3 个月变成 2 3 年。激光雕刻陶瓷 网纹辊推动柔性版印刷术发生了一次新的革命, 是激光技 术的一次很成功的应用。

由于声光调 $\mathrm{Q}$ 的 $\mathrm{YAG}$ 激光采用电信号控制光脉冲, 可控性好; 其波长是 $\mathrm{CO}_{2}$ 激光的 $1 / 10$, 可以聚焦到更小的 光斑尺寸; 陶瓷对 $\mathrm{YAG}$ 激光的吸收率也比 $\mathrm{CO}_{2}$ 激光更 高。故 YAG 激光比 $\mathrm{CO}_{2}$ 激光更适合于雕刻高线数、高质 量的陶瓷网纹辊。1996 年世界上首次出现 YAG 激光雕刻 陶瓷网纹辊 ${ }^{[3]}$, 目前采用光纤激光器的 YAG 激光雕刻陶 瓷网纹辊的雕刻线数最高可达 4000 线/英寸 ${ }^{[4]}$, 为在柔性
版印刷中实现高精细印刷创造了条件。研发 YAG 激光雕 刻陶瓷网纹辊技术意义重大。

YAG 激光毛化轧辊技术是中国科学院力学研究所近 20 年逐步研发的集激光技术、声光调制技术、控制技术、 计算机技术、材料表面处理技术等先进技术于一体的具有 自主知识产权的先进的激光加工技术体系。“YAG 激光 毛化轧辊技术及应用” 获得了 2007 年度国家技术发明二 等奖。中科院力学所成功地将 YAG 激光毛化轧辊技术用 于雕刻陶瓷网纹辊, 研制出国内第一台具有自主知识产权

的 YAG 激光雕刻陶瓷网纹辊设备, 填补了国内空白。

本文将介绍 YAG 激光雕刻陶瓷网纹辊的几项关键技 术。

\section{YAG 激光雕刻陶瓷网纹辊加工工艺}

\section{A. 加工过程}

(1)辊体预加工。网纹辊的辊体一般选用优质碳素钢 管, 预加工包括粗加工 $\rightarrow$ 动平衡 $\rightarrow$ 轴与法兰盘加工 $\rightarrow$ 焊接 $\rightarrow$ 机械加工 $\rightarrow$ 调质处理 $\rightarrow$ 半精加工 $\rightarrow$ 精加工。

(2)等离子喷涂。用等离子喷涂的方法，将金属氧化物 $\left(\mathrm{Al}_{2} \mathrm{O}_{3}\right.$ 或 $\left.\mathrm{Cr}_{2} \mathrm{O}_{3}\right)$ 熔化, 熔射涂布在金属光辊表面, 形成 高硬度、与金属辊结合牢固、致密的陶瓷薄膜。

(3)研磨、抛光。

(4)激光雕刻。用经过特殊调制的高能量密度 $\left(10^{4} \sim 10^{6}\right.$ $\mathrm{W} / \mathrm{cm}^{2}$ ) , 高重复频率 (每秒数千至数万次) 的 YAG 激 光脉冲, 聚焦照射到旋转的陶瓷辊表面, 再辅以一定成 份, 一定压力的辅助气体, 沿光束同轴方向吹熔池中的熔 融物, 形成具有一定形貌的网穴结构。与此同时, 脉冲激 光束以一定速度沿陶瓷辊轴向匀速移动, 在整个辊面形成 一定分布的大量网穴。

\section{B. 单个网穴要求}

(1)直径可变, 且变化范围大, 以适应不同线数的要 求。

(2)深度可变, 且变化范围大, 以适应不同的载墨量。

(3)网穴表面结构有利于油墨的储存和传递。

\section{C. 网穴分布要求}


网穴分布有 $30^{\circ}$ 六角形、 $45^{\circ}$ 四角形和 $60^{\circ}$ 六角形三种 规格。图 1 是陶瓷网纹辊网穴分布示意图, 从左到右分别 是 $30^{\circ}$ 六角形、 $45^{\circ}$ 四角形和 $60^{\circ}$ 六角形的示意图。目前应 用最多的是 $60^{\circ}$ 六角形的网穴, 已成了行业标准。
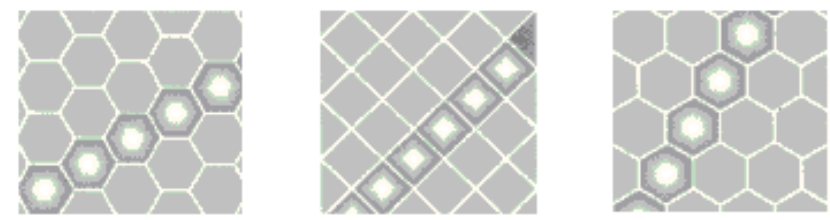

图 1 陶瓷网纹辊网穴分布示意图

Fig. 1 Sketch of ceramic anilox' cell distribution

D. 技术难点

(1)直径、深度可变的单网穴的雕刻问题。需解决高能 量激光和陶瓷的能量耦合问题, 提高陶瓷对激光的吸收效 率。

(2)网穴的高精度定位问题。高线数的陶瓷网纹辊的网 墙很窄 (几微米), 故对网穴的定位精度要求很高。

(3)网穴分布问题。实现 $30^{\circ}$ 六角形、 $45^{\circ}$ 四角形和 $60^{\circ}$ 六角形三种规格。

\section{III. 关键技术}

\section{A. 高重频光脉冲波形调制技术}

传统声光调 $\mathrm{Q}$ 光脉冲, 脉宽较窄（小于 $1 \mu \mathrm{s}$ ) 且不可 控, 能量密度高, 雕刻凹坑浅, 生产效率低, 使之无法用 于工业化生产。中科院力学所从理论上解释了出现以上现 象的主要原因: 当传统声光调 $\mathrm{Q}$ 光脉冲作用到辊面时瞬时 发生气化, 激光所产生的强烈等离子体羽对后续激光能量 产生强烈的吸收和反射, 屏蔽了后续光能量的耦合 ${ }^{[5]}$ 。据 此研发了高重频光脉冲波形调制技术。图 2 是 YAG 激光 毛化工作范围。

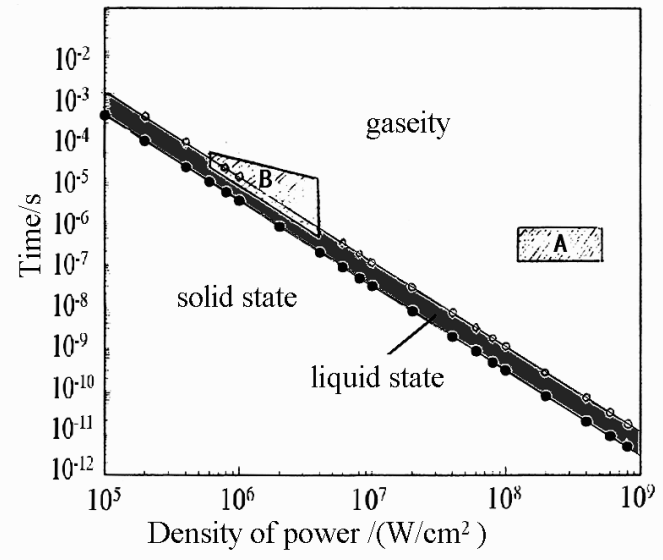

图 2 YAG 激光毛化工作范围

Fig. 2 Sketch of area using in YAG laser texturing

将激光脉冲工作点参数从 A 区 (气化区) 调到 B 区 （气化一熔化区），抑制气化, 增加熔化。在微秒级时间 间隔内, 将常规声光调 $\mathrm{Q}$ 的阶跃式开关变成多段斜率渐变 式超声场调制, 即 “轻调 $\mathrm{Q}$ ” ${ }^{[6]}$, 来调控激光腔增益, 实 现展宽光脉冲、压制峰值功率，既让激光脉冲有足够的能
量使辊面毛化点气化一熔化, 又可使波形能量密度随时间 分布与辊面升温后吸收率变化趋势相一致, 提高光能量耦 合效率。

图 3 是常规调制光波形图。图 4 是波形调制光波形 图。波形调制与常规调制相比, 在相同脉冲能量条件下, 毛化粗糙度提高了 1.5 倍, 同时毛化速度提高了 1 倍。高 重频光脉冲波形调制技术是 YAG 激光毛化技术中的一项 核心技术，在推广应用中起到关键作用。

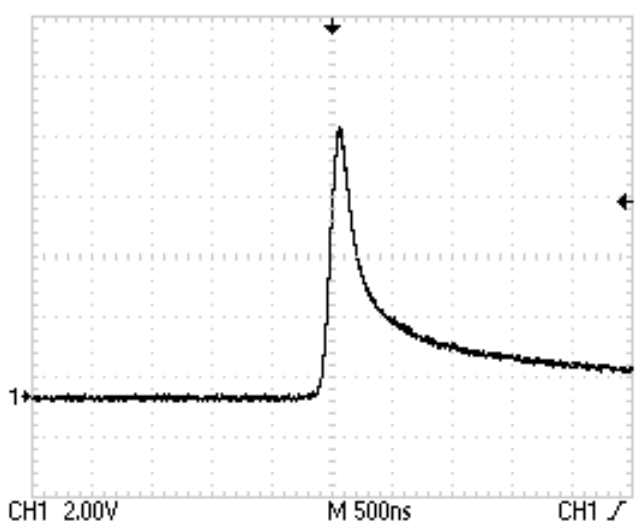

图 3 常规调制光波形图

Fig. 3 Sketche of light pulse modulated generally

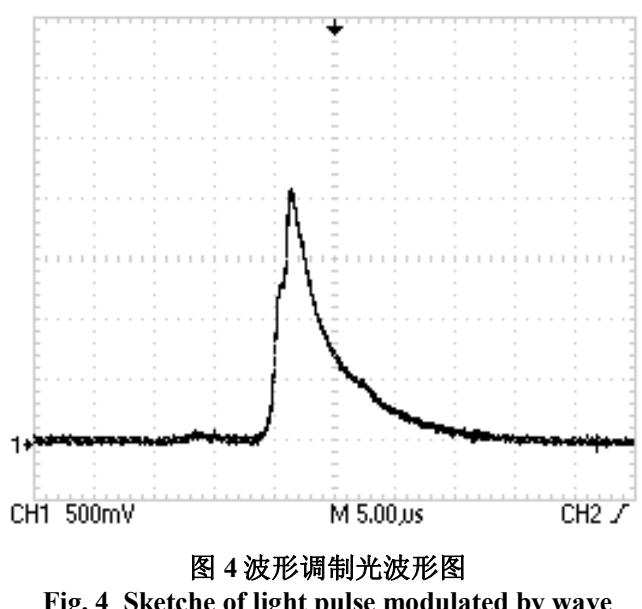

高重频光脉冲波形调制技术也是 YAG 激光雕刻陶瓷 网纹辊技术中的关键技术之一, 利用它可雕出较理想的网 穴。该技术在 YAG 激光雕刻陶瓷网纹辊中的应用与在 $Y A G$ 激光毛化中的应用也有区别。在 YAG 激光毛化中, 因需要凸台, 激光作用以熔化为主。在 YAG 激光雕刻陶 瓷网纹辊中, 因不希望有凸台, 激光作用以气化为主。

在实际应用中, 光脉冲波形是一种特殊的工艺参数, 将它与激光器平均功率、雕刻频率等其他工艺参数相配 合, 可雕出不同直径、不同深度的网穴, 以满足不同线 数、不同载墨量的雕刻要求。

\section{B. 二维可控分布技术}

利用 YAG 激光毛化由控制声光电源的电信号控制激 光器发出光脉冲, 响应速度很快, 光脉冲只滞后电信号几 
微秒的特点, 通过同轴安装的检测装置（高精度高分辨率 编码器) 测量轧辊的位置, 用工控机通过可控分布接口卡 和可控分布软件对检测信号在线变分频处理, 产生控制声 光电源的电信号, 声光电源控制激光器发出光脉冲, 精确 控制轧辊毛化点的圆周向间距，同时以电子齿轮方式通过 机床丝杜驱动聚焦头轴向移动精确控制毛化点的轴向间 距，从而实现二维可控制分布 ${ }^{[7][8]}$ 。文献 ${ }^{[7]}$ 已证明该技术在 圆周向可实现很高的定位精度，图 5 是二维可控分布技术 示意图。二维可控分布技术可实现矩形、菱形两种分布, 矩形分布可用于图形（图像）雕刻，菱形分布可用于 YAG 激光雕刻陶瓷网纹辊中的网穴雕刻, 满足网穴分布 的要求。

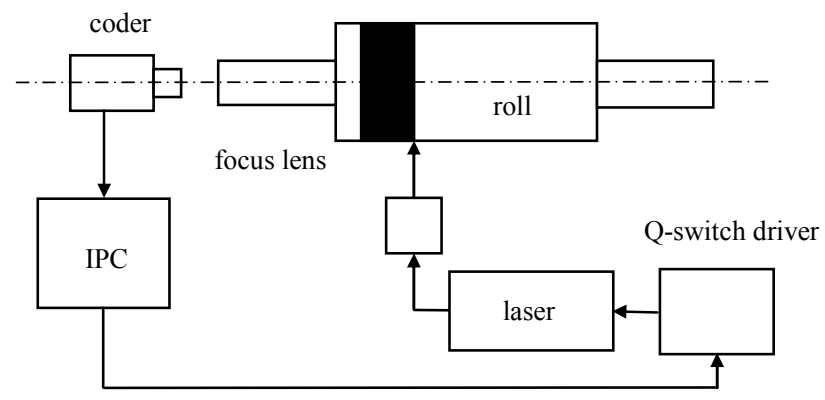

图 5 二维可控分布技术示意图

Fig. 5 Sketch of tow dimension controllable distribution technology

为了加快雕刻速度，一般采用连续螺旋线雕刻方式。 激光器发出光脉冲雕出网穴, 聚焦头轴向恒速移动, 顺序 产生的网穴形成一条螺旋线。将编码器一转的脉冲数等分 成奇数份, 相当于将辊面等分成奇数份。工控机对编码器 的脉冲计数, 每计到相连两份位置产生电信号, 控制激光 器发出光脉冲, 就可雕出具有菱形分布的网穴。图 6 是菱 形分布展开示意图。

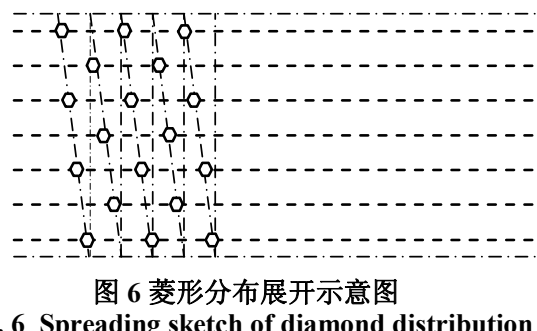

Fig. 6 Spreading sketch of diamond distribution

点脉冲数 (雕刻相邻两个微坑之间编码器输出的脉冲 数) $\mathrm{J}$, 转点数 (辊面等分数) $\mathrm{Z}$ 和余脉冲数 $\mathrm{C}$ 分别为:

$$
\begin{aligned}
& \mathrm{J}=[\mathrm{dM} / \mathrm{D} \pi] \\
& \mathrm{Z}=[\mathrm{M} / \mathrm{J}] \\
& \mathrm{C}=\mathrm{M}-\mathrm{ZJ}
\end{aligned}
$$

如果 $Z$ 为偶数, $Z$ 减 1 , 保证其为奇数。式中 $D$ 为辊 直径, $\tau$ 为螺距, $d$ 为点距 (辊面等分间距), $M$ 为编码 器一转输出的脉冲数。

为了将辊面等分尽量均匀, 将 $\mathrm{C}$ 个余脉冲作均布处 理, 用公式表示为:

$$
\mathrm{M}=\mathrm{C}(\mathrm{J}+1)+(\mathrm{Z}-\mathrm{C}) \mathrm{J}
$$

即辊面一周有 $\mathrm{C}$ 个等分间距相当于 $\mathrm{J}+1$ 个脉冲，有 $\mathrm{Z}-\mathrm{C}$ 个等分间距相当于 $\mathrm{J}$ 个脉冲, 每 $[\mathrm{Z} / \mathrm{C}]$ 个等分间距中包 含一个相当于 $\mathrm{J}+1$ 个脉冲的等分间距。这种方法的辊面等 分间距的误差为 $1 / \mathrm{J}$, 只要 $\mathrm{J}$ 足够大，辊面等分间距的误差 可控制在要求的范围内。如果余脉冲在辊面一周的同一位 置分配, 而 $\mathrm{J}$ 又不够大, 将会在辊面轴向出现 $\mathrm{C}$ 条与辊面 轴线平行的直线, 这种陶瓷网纹辊实际中无法使用。为了 解决这问题，采用了无规则均分的方法，也即每 $[\mathrm{Z} / \mathrm{C}]$ 个等 分间距中包含一个相当于 $\mathrm{J}+1$ 个脉冲的等分间距, 其位置 在此范围内是无规则分布的。图 7 是控制脉冲时序示意 图。为此, 需要采用一种变分频技术。工控机需对编码器 信号在线变分频处理, 也即辊每转一周, 工控机要作 C 次 $\mathrm{J}+1$ 计数和 $\mathrm{Z}-\mathrm{C}$ 次 $\mathrm{J}$ 计数, 两种计数在一定范围内无规则 交替进行。

由于螺距与辊的周长相比很小, 可认为螺旋线与辊的 轴线垂直。通过点距和螺距的预先设定就可实现 $30^{\circ}$ 六角 形、 $45^{\circ}$ 四角形和 $60^{\circ}$ 六角形三种分布:

当 $\mathrm{d}=\tau / 1.732$ 时, 为 $30^{\circ}$ 六角形分布;

当 $\mathrm{d}=\tau$ 时, 为 $45^{\circ}$ 四角形分布;

当 $\mathrm{d}=1.732 \tau$ 时, 为 $60^{\circ}$ 六角分布。

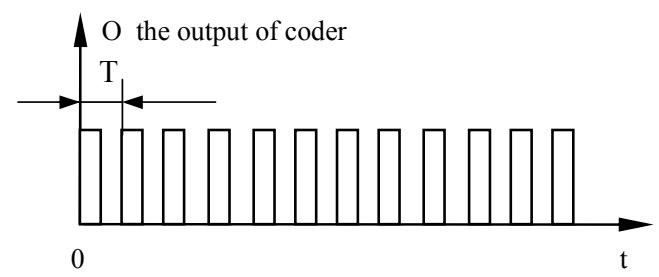

O1 the control singal of Q-switch driver

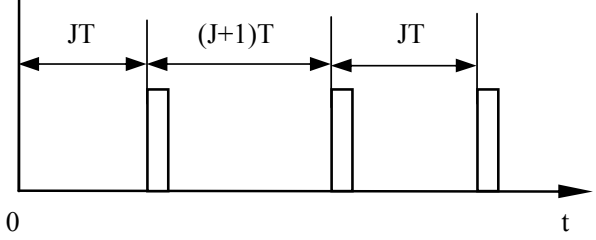

图 7 控制脉冲时序示意图

Fig. 7 Timing sketch of control pulses

\section{C. 高重频编组脉冲技术}

由于受到调制器关断功率限制, 用声光调 $Q$ 的 YAG 激光器的功率比较小, 最大几百瓦, 雕刻低线数, 高载墨 量的网纹辊时用单个光脉冲一次雕出单个网穴无法实现。 通过编组电脉冲信号控制声光电源, 使激光器发出一组光 脉冲; 通过设置脉冲个数和频率, 实现高深度雕刻 ${ }^{[6]}$ 。

组脉冲频率比网穴雕刻频率高很多 (如组脉冲频率为 $40 \mathrm{kHz}$ ，网穴雕刻频率为 $2 \mathrm{kHz}$ ），以致当一组脉冲作用 在辊面上时, 这些脉冲基本上作用于同一网穴上。组脉冲 内的脉冲数可以根据需要设置, 脉冲数越多, 雕出的网穴 越深。图 8 是高重频编组脉冲时序图。

英国提供的 YAG 激光雕刻陶瓷网纹辊设备装备了多 次击打同一网穴的雕刻技术，第一次脉冲形成网穴，而后 几次脉冲可用来增加网穴容积 ${ }^{[4]}$ 。高重频编组脉冲技术在 YAG 激光雕刻陶瓷网纹辊中起到了类似的作用。 
(a)

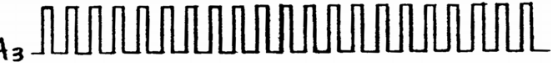

(b)
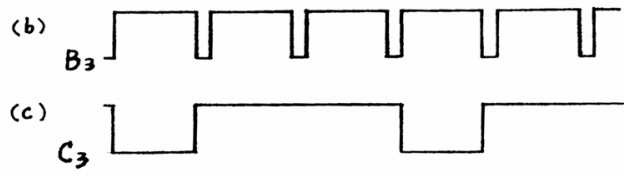

(d) $\bar{c}_{3}$

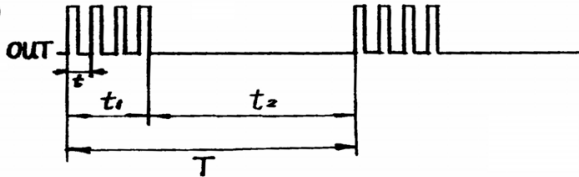

图 8 高重频编组脉冲时序图

Fig. 8 Timing sketch of high repetition and grouped pulses

在实际应用中发现, 用组脉冲雕出的网穴比单脉冲雕 出的网穴更光滑, 更有利于油墨的传递。

\section{IV. 结论}

高重频光脉冲波形调制技术通过将激光腔增益调控方 式由开关式变成渐变式解决了陶瓷对 YAG 激光的吸收效 率问题; 高重频编组脉冲技术通过一组光脉冲作用于同一 网穴解决了高深度网穴的雕刻问题; 二维可控分布技术通 过编码器检测和变分频信号处理解决了网穴定位和网穴分 布问题。为了实现不同线数不同载墨量的雕刻, 以上三项 YAG 激光毛化技术缺一不可, 它们是实现 YAG 激光雕刻 网纹辊的关键技术。用以上关键技术制造的 YAG 激光雕 刻网纹辊设备已在国内得到成功应用, 效果良好。图 9 是 陶瓷网纹辊 $60^{\circ}$ 六角形网穴雕刻图。

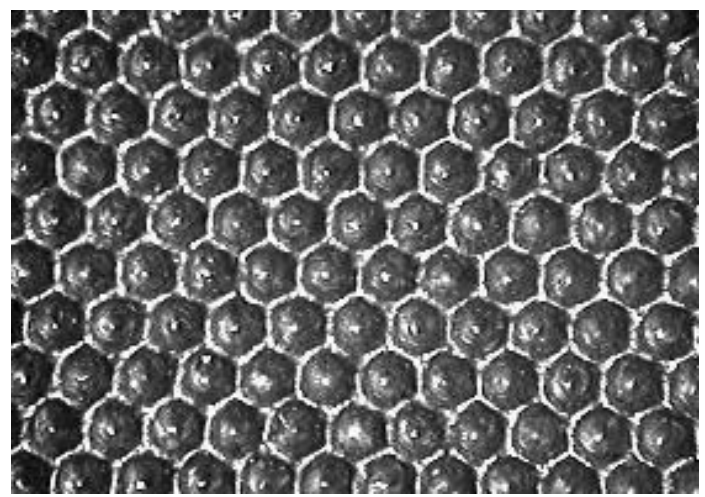

图 9 陶瓷网纹辊 $60^{\circ}$ 六角形网穴雕刻图

Fig. 9 Carving sketch of ceramic anilox' $60^{\circ}$-hexagon cell distribution

用高重频编组脉冲技术雕刻的高深度网穴存在缺陷。 因网穴是椭圆的，不美观，影响网穴网墙尺寸; 椭圆长轴 两端较浅, 影响载墨量; 有时为了保证墨穴的几何尺寸， 只能降低雕刻频率，降低雕刻效率。故需要研发更先进的 技术。

\section{致谢}

本论文获国家重点科技攻关项目一一 “冷轧薄钢板激 光毛化（95-B11-01-01）”和中国科学院重大项目 “YAG 激光毛化技术深度研究开发（KY951-A1-601）”的子课 题——“光电复合毛化及可控毛化新技术” 的支持。

\section{REFERENCES}

[1] Yinhe Jin, Flexography, Chemical Industry Press, 2001 (in Chinese).

[2] Siqi Zou, "Anilox Roller:Heart of Flexographic Press," Print Word, 2004, 30(3): 9-11(in Chinese).

[3] Zaitong Lu, "Development of Laser Engraving Technique," Pinting Fleid, 2001, 182(5):51-54(in Chinese).

[4] Haojie Chen, Shangkun Yang, and Lili Han, "On Flexo Printing Anilox Roll Technology Development," Print Today, 2007, 25(3):26-27(in Chinese).

[5] Mingjiang Yang, Linhua Peng, and Zhengyang Li, "Development of YAG Laser Texturing Technology," Applied Laser, 2002, 22(3): 323326(in Chinese).

[6] Mingjiang Yang, Guangnan Chen, Jian Wu et al.. High Repetition, Multi-pulsed YAG Laser Engraving System and its Application in Laser Texturing Rolls, China Talent, ZL92113223.9, 1995.07.08(in Chinese).

[7] Hongcai Wang, Mingjiang Yang, "A Numerical Control System of Controllable Distribution on YAG Laser-texturing Equipment," Computer Automated Measurement \& Control, 2001, 9(3):11-13(in Chinese).

[8] Hongcai Wang, Mingjiang Yang, A Laser Processing System for the Texturing Roller Surface with Controllable Micro-criters, China Talent, ZL00128273.5, 2004.06.02(in Chinese). 